\title{
Performance Comparison of Power Quality Evaluation Using Advanced High Resolution Spectrum Estimation Methods
}

\author{
Md. Ziaul Hoque ${ }^{1}$, Md. Raju Ahmed ${ }^{2}$ \\ ${ }^{1}$ Research Scholar, ${ }^{2}$ Professor \\ Department of Electrical and Electronic Engineering \\ Dhaka University of Engineering and Technology, Bangladesh
}

\begin{abstract}
Most of the conventional methods of power quality assesment in power systems are almost exclusively based on Fourier Transform that suffer from various inherent limitations. First limitation of an FFT based method is that of frequency resolution, whereas the second limitation is due to no coherent signal sampling of the data which proves itself as a leakage in spectrum domain. These two performance limitations of FFT or similar methods are particularly troublesome when analyzing short data records. To overcome from this problem, high regulation spectrum estimation methods can be used where resolution problem is not found. In this thesis, high resolution methods, such as MUSIC, root MUSIC and ESPRIT are discussed that use a different approach to spectral estimation; instead of trying to estimate the power spectral density (PSD) directly from the data, they model the data as the output of a linear system driven by white noise, and then attempt to estimate the parameters of that linear system. Detail Matlab simulations are carried out in order to investigate the performance of MUSIC, Root MUSIC and ESPRIT methods in estimating amplitude, power (squared amplitude) and frequency estimation of synthetic power signal both in clean and noisy conditions. Using mean square error (MSE) as the evaluation criterion, the variation of amplitude, power (squared amplitude) and frequency estimation are shown with respect to data sequence length and SNR and their influences on MSE are compared for the different methods as mentioned above.
\end{abstract}

Keywords: Conventional Methods, Parametric \& Nonparametric Methods, High Resolution Spectral Methods, MUSIC, Root MUSIC and ESPRIT, Data Length, Signal to Noise Ratio, Means Square Error.

\section{Introduction}

Now a days, the quality of power or voltage waveforms has become a matter of great importance for power utilities, electrical energy customers and additionally for manufacturers of electrical and electronic equipment. The energy markets will strengthen the competition and is expected to drive down the prices of energy which is the main part for the requirements concerning the power quality. Normally the power and voltage waveform is expected to be a pure sinusoidal with a given amplitude and frequency. Most of the fashionable frequency power converters generate a large spectrum of harmonic elements that indicate the standard of the delivered energy and increase the energy. The proliferation of nonlinear loads connected to power systems has triggered a growing concern with power quality issues. The inherent operation characteristics of these loads deteriorate the quality of the delivered energy, and increase the energy losses as well as decrease the reliability of a power system [1], [2], [6]. In some cases, large convertor systems generate not solely characteristic harmonics typical for the perfect convertor operation however additionally a substantial quantity of non characteristic harmonics and inter harmonics, which can powerfully verify the standard of the powersupply voltage [7], [8]. So, that higher management control and protection depend on the estimation of the signal elements. Normally Power could be defined as the actual physical power, or more often, for convenience with abstract signals, could be defined as the squared value of the signals. (Statisticians study the variance of a set of data, but because of the analogy with electrical signals, still refer to it as the power spectrum).The total power of the signals would be a time average since power has units of energy/time.

$$
\lim _{T \rightarrow \infty} \frac{1}{2 T}{ }_{-T}^{T} x(t)^{2} d t
$$

Also the power of a signal may be finite even if the energy is infinite. Power also could be defined as the squired amplitude of a particular signal.

Where $A_{i}$ is the amplitude of a particular signal.

$$
\mathrm{P}=\frac{1}{\mathrm{~N}} \sum_{\mathrm{i}=1}^{\mathrm{N}} \mathrm{A}_{\mathrm{i}}^{2}
$$

In power system frequency, amplitude and phasor are the most important parameters for monitoring, control and protection. All of these can reflect the whole power system situation. In electrical power system it is of utmost important to keep the frequency as close to its original value as possible. In order to control the power 
system frequency it needs to be measured quickly and accurately. Normally power system voltage and current waveforms are distorted by harmonic and inter harmonic components, particularly during system disturbance. Faults or other switching transients may change the magnitude and phase angles of the waveforms. However, voltage and current can also be distorted by non-linear loads, power electronic components and inherent nonlinear nature of the system elements [8]. Not only that the assessment of power quality can be done either by calculating, measuring or estimating power quality indices (frequency, spectrum, harmonic distortion etc.). So that the estimation of power quality indices is still an important and yet challenging part in power system. Only the estimation techniques can improve the accuracy of measurement of spectral parameters of distorted waveforms encountered in power systems, in particular the estimation of the power quality indices [9]. More reliable methods are required for power quality monitoring and estimation.

\section{The Proposed Methods}

There are various kinds of methods that are used in power, amplitude and frequency estimation to improve the accuracy of measurement of spectral parameters of distorted waveforms encountered in power systems, in particular the estimation of the power quality indices but the conventional high resolution methods are more effective. In this chapter, the description of the methods of high resolution especially MUSIC, ESPRIT and ROOT MUSIC are provided.

\section{A. MUSIC Method}

The idea of MUSIC (Multiple Signal Classification) was developed in [15] where the averaging was proposed for improvement of the performance of Pisarenko estimator [16]. Instead of using only one noise eigenvector, the MUSIC method uses many noises Eigen filters. The number of computed eigen values $M>K$ +1 . MUSIC method is a method which presents signal sub space and using this method we can get more accurate result than normal methods.

Let a signal to describe the MUSIC method.

$$
x=\sum_{i=1}^{p} A_{i} s_{i}+\eta ; A_{i}=\left|A_{i}\right| e^{j \varphi_{i}}
$$

Where $\mathrm{S}_{\mathrm{i}}=\left[1 \mathrm{e}^{\mathrm{j} \omega_{1 \ldots . . .} \mathrm{e}^{\mathrm{j}(\mathrm{N}-1) \omega_{2}}}\right]^{\mathrm{T}} \quad, A_{i}$-amplitudes of the signal components, N-number of signal samples, $\mathrm{p}$ number of components, $\mathrm{y}$-noise, $\varphi_{i}$-components frequency;

Now let a signal samples to estimate autocorrelation matrix as:

$$
\mathrm{R}_{\mathrm{x}}=\sum_{\mathrm{i}=1}^{\mathrm{p}} \mathrm{E}\left\{\mathrm{A}_{\mathrm{i}} \mathrm{A}_{\mathrm{i}}^{*} S_{\mathrm{i}} \mathrm{S}_{\mathrm{i}}^{\mathrm{T}}+\sigma_{0}^{2} \mathrm{I}\right.
$$

$N-p$ smallest eigen values of the correlation matrix (matrix dimension $N>p+1$ ) correspond to the noise subspace and $p$ largest (all greater than $\sigma_{0}^{2}$ - noise variance) correspond to the signal sub space.

The matrix of noise eigenvectors of the above matrix (2) is used

$$
\mathrm{E}_{\text {noise }}=\left[\begin{array}{llll}
\mathrm{e}_{\mathrm{p}+1} & \mathrm{e}_{\mathrm{p}+2} & \cdots & \ldots \\
\mathrm{N}
\end{array}\right]
$$

to compute the projection matrix for the noise subspace:

$$
\mathrm{P}_{\text {noise }}=\mathrm{E}_{\text {noise }} \mathrm{E}_{\text {noise }}^{* \mathrm{~T}}
$$

Which, by using an auxiliary vector $\mathrm{W}=\left[\begin{array}{ll}1 & \mathrm{e}^{\mathrm{j} \omega_{\mathrm{t}}} \ldots . . . \mathrm{e}^{\mathrm{j}(\mathrm{N}-1) \omega_{\mathrm{t}}}\end{array}\right]^{\mathrm{T}}$ allows computation of projection of vector, $\mathrm{W}$ onto the noise subspace as:

$$
\begin{aligned}
\mathrm{w}^{* \mathrm{~T}} \mathrm{P}_{\text {noise }} \mathrm{w} & =\mathrm{w}^{* \mathrm{~T}} \mathrm{E}_{\text {noise }} \mathrm{E}_{\text {noise }}^{* \mathrm{~T}} \mathrm{w}= \\
& =\sum_{\mathrm{i}-\mathrm{p}+1}^{\mathrm{N}} \mathrm{E}_{\mathrm{i}}\left(\mathrm{e}^{\mathrm{j} \omega}\right) \mathrm{E}_{\mathrm{i}}^{*}\left(\mathrm{e}^{\mathrm{j} \omega}\right) \stackrel{\mathrm{z}}{\rightarrow} \sum_{\mathrm{i}-\mathrm{p}+1}^{\mathrm{N}} \mathrm{E}_{\mathrm{i}}(\mathrm{z}) \mathrm{E}_{\mathrm{i}}^{*}\left(1 / \mathrm{z}^{*}\right)
\end{aligned}
$$

The last polynomial in (5) has p double roots lying on the unit circle, which angular positions correspond to the frequencies of the signal components. This method of finding the frequencies is therefore called root-MUSIC. After the calculation of the frequencies, the powers of each component can be estimated from the eigen values and eigenvectors of the correlation matrix, using the relations

And solving for $\mathrm{p}_{\mathrm{i}}-$ components power.

$$
\mathrm{e}_{\mathrm{i}}^{* \mathrm{~T}} \mathrm{R}_{\mathrm{x}} \mathrm{e}_{\mathrm{i}}=\lambda_{\mathrm{i}} \text { and } \mathrm{R}_{\mathrm{x}}=\sum_{\mathrm{i}=1}^{\mathrm{p}} \mathrm{P}_{\mathrm{i}} \mathrm{s}_{\mathrm{i}} \mathrm{s}_{\mathrm{i}}^{* \mathrm{~T}}+\sigma_{0}^{2} \mathrm{I}
$$

\section{B. Root MUSIC Method}

Root-MUSIC method facilitates the same ideas with MUSIC and differs only in the second step of the MUSIC algorithm. The main advantage of Root-MUSIC over MUSIC is its lower computational complexity. The MUSIC spectrum is an all pole function of the form

Let $\mathrm{C}=\mathrm{U}_{\mathrm{N}} \mathrm{U}_{\mathrm{N}}^{\mathrm{H}}$ using equation (7) written as

$$
\mathrm{P}_{\mathrm{mu}}(\theta)=\frac{1}{\operatorname{abs}\left[\mathrm{F}(\theta)^{\mathrm{H}} \mathrm{U}_{\mathrm{N}} \mathrm{U}_{\mathrm{N}}^{\mathrm{H}} \mathrm{F}(\theta)\right]}
$$

$$
\mathrm{P}_{\mathrm{mu}}^{-1}=\sum_{\mathrm{m}=1}^{\mathrm{M}} \sum_{\mathrm{n}=1}^{\mathrm{M}} \exp \left(\frac{\mathrm{j}(\mathrm{m}-1) 2 \pi \mathrm{d} \sin \left(\theta_{\mathrm{b}}\right)}{\lambda}\right) \mathrm{C}_{\mathrm{mn}} \mathrm{A}
$$


Where $\quad A=\exp \left(\frac{-j(m-1) 2 \pi d s i n\left(\theta_{b}\right)}{\lambda}\right)$, and $C_{m n}$ is the entry in the $m^{t h}$ row and $n^{\text {th }}$ column of C. Combination of two sums into one gives equation (9):

$$
\mathrm{P}_{\mathrm{mu}}^{-1}=\sum_{\mathrm{n}=1}^{\mathrm{M}} \mathrm{C}_{1} \exp \left(\frac{-\mathrm{j} 2 \pi \mathrm{d} \mathrm{l} \sin \left(\theta_{\mathrm{b}}\right)}{\lambda}\right)
$$

Where $\mathrm{C}_{1}=\sum_{\mathrm{m}-\mathrm{n}=\mathrm{l}} \mathrm{C}_{\mathrm{mn}}$ is the sum of the entries of $\mathrm{C}$. Along the $l^{\text {th }}$ diagonal polynomial representation $\mathrm{D}(\mathrm{z})$ will

$$
\mathrm{D}(\mathrm{z})=\sum_{\mathrm{l}=-\mathrm{M}+1}^{\mathrm{M}+1} \mathrm{C}_{1} \mathrm{z}^{-1}
$$

If the eigen decomposition corresponds to the true spectral matrix, then MUSIC spectrum becomes equivalent to the polynomial $\mathrm{D}(\mathrm{z})$ on the unit circle and peaks in the MUSIC spectrum exists as ROOTs of the D(z) lie close to the unit circle [17]. A pole of $\mathrm{D}(\mathrm{z})$ at $\mathrm{z}=z_{1}=\left|z_{1}\right| \exp \left(\mathrm{jarg}\left(z_{1}\right)\right.$ ) will result in a peak in the MUSIC spectrum at $\theta=\sin ^{-1}\left(\{\lambda / 2 \mathrm{rd}\} \arg \left[\mathrm{z}_{1}\right]\right)$.

\section{ESPRIT Method}

The original ESPRIT (Estimation of Signal Parameter via Rotational Invariance Technique) was developed by another one as in example [13].It is based on a naturally existing shift invariance between the discrete time series which leads to rotational invariance between the corresponding signal subspaces. The shift invariance is illustrated below. After the Eigen-decomposition of the autocorrelation matrix as:

$$
\mathrm{R}_{\mathrm{x}}=\mathrm{U}^{* \mathrm{~T}} \mathrm{~A}
$$

It is possible to partition a matrix by using special selector matrices which select the first and the last $(M-1)$ columns of a $(M \times M)$ matrix, respectively:

$$
\begin{aligned}
& \Gamma_{1}=\left[\mathrm{I}_{\mathrm{M}-1} \mid 0_{(\mathrm{M}-1) \times 1}\right]_{(\mathrm{M}-1) \times \mathrm{M}} \\
& \Gamma_{1}=\left[0_{(\mathrm{M}-1) \times 1} \mid \mathrm{I}_{\mathrm{M}-1}\right]_{(\mathrm{M}-1) \times \mathrm{M}}
\end{aligned}
$$

By using of matrices $\Gamma$ two subspaces are defined, spanned by two subsets of eigenvectors as follows:

$$
\begin{aligned}
& \mathrm{S}_{1}=\Gamma_{1} \mathrm{U} \\
& \mathrm{S}_{2}=\Gamma_{2} \mathrm{U}
\end{aligned}
$$

For the matrices defined as $S_{1}$ and $S_{2}$ in (13), for every $\omega_{k} ; K \epsilon N$, representing different frequency components, and matrix $\phi$, defined as:

$$
\Phi=\left[\begin{array}{cccc}
\mathrm{e}^{\mathrm{j} \omega 1} & 0 & \cdots & 0 \\
0 & \mathrm{e}^{\mathrm{j} \omega 2} & 0 & 0 \\
\vdots & \vdots & \vdots & \vdots \\
0 & 0 & \cdots & \mathrm{e}^{\mathrm{j} \omega \mathrm{k}}
\end{array}\right]
$$

The following relation can be proven [14]:

$$
\left[\Gamma_{1} \mathrm{U}\right] \phi=\Gamma_{2}
$$

The matrix $\phi$ contains all information about frequency components. In order to extract this information, it is necessary to solve (15) for $\phi$. By using a unitary matrix (denoted as T), the following equations can be derived:

$$
\begin{aligned}
& \Gamma_{1}(\mathrm{UT}) \phi=\Gamma_{2}(\mathrm{UT}) \\
& \left.\Gamma_{1} \mathrm{U}^{\mathrm{T}} \phi \mathrm{T}^{* \mathrm{~T}}\right)=\Gamma_{2}
\end{aligned}
$$

In the further considerations the only interesting subspace is the signal subspace, spanned by signal eigenvectors $U_{s}$. Usually it is assumed that these eigenvectors correspond to the largest Eigen values of the correlation matrix and $\mathrm{U}_{\mathrm{s}}=\left[\mathrm{u}_{1}, \mathrm{u}_{2}, \ldots \ldots \ldots, \mathrm{u}_{\mathrm{k}}\right]$. ESPRIT algorithm determines the frequencies $e^{j \omega k}$ as the eigenvalues of the matrix $\phi$. In theory, the equation (15) is satisfied exactly. In practice, matrices $S_{1}$ and $S_{1}$ are derived from an estimated correlation matrix, so this equation does not hold exactly, it means that (15) represents an over-determined set of linear equations.

\section{Simulation Results And Performance Comparison}

\section{A. Simulation Conditions}

> Signal $\quad \mathrm{y}=.99 * \sin (2 * \pi * \mathrm{t} * 100)+.97 * \sin (2 * \pi * \mathrm{t} * 150)$ is used.

> Random noise is generated by using randn function in Matlab.

>> Sampling frequency $50<$ Fs $<1000 \mathrm{~Hz}$.

> SNR varies from 100 to $0 \mathrm{~dB}$.

> Signal length from 50 to 400 samples is considered.

\section{B. Performance Evaluation Criterion}

The criterion used for evaluating the MUSIC, ESPRIT and Root MUSIC is mean square error (MSE) with respect to data length and SNR. The equation of MSE is given by, 
For amplitude estimation, $\mathrm{A}_{\mathrm{MSE}}=\sqrt{ }\left[\left(\frac{1}{\mathrm{~N}}\right) \sum_{\mathrm{i}=1}^{\mathrm{N}}\left(\mathrm{A}_{\text {iest }}-\mathrm{A}_{\text {org }}\right)^{2}\right]$.

For Frequency estimation, $\mathrm{F}_{\mathrm{MSE}}=\sqrt{ }\left[\left(\frac{1}{\mathrm{~N}}\right) \sum_{\mathrm{i}=1}^{\mathrm{N}}\left(\mathrm{f}_{\text {iest }}-\mathrm{f}_{\text {org }}\right)^{2}\right]$.

And for power estimation, $\left.\mathrm{P}_{\mathrm{MSE}}=\sqrt{[}\left(\frac{1}{\mathrm{~N}}\right) \sum_{\mathrm{i}=1}^{\mathrm{N}}\left(\mathrm{A}_{\text {iest }}{ }^{2}-\mathrm{A}_{\text {org }}{ }^{2}\right)\right]$.

Where

$A_{\text {iest }}=$ Estimated amplitude

$\mathrm{A}_{\text {org }}=$ Original amplitude

$\mathrm{f}_{\text {iest }}=$ Estimated frequency

$\mathrm{f}_{\text {org }}=$ Original frequency

\section{Amplitude Estimation}

Amplitude is the most important parameter in power system monitoring, control, and protection. It can reflect the whole power system situation. In electrical power system it is of utmost importance to keep the amplitude as close to its nominal value as possible. In order to control the power system amplitude it needs to be measured quickly and accurately. For this the estimation of amplitude is still an important and yet challenging part. The effects of data length and SNR on MSE for amplitude estimation are following:

\section{Power Estimation}

In general, power system voltage and current waveforms are distorted by harmonic and inter harmonic components, particularly during system disturbance. Faults or other switching transients may change the magnitude and phase angles of the waveforms. However, voltage and current can also be distorted by non-linear loads, power electronic components and inherent non-linear nature of the system elements [3].Not only that the assessment of power quality can be done either by calculating, measuring or estimating power quality indices (frequency, spectrum, harmonic distortion etc.).Only the estimation techniques can improve the accuracy of measurement of spectral parameters of distorted waveforms encountered in power systems, in particular the estimation of the power quality indices [4].For these reason the power estimation is very important. The effects of data length and SNR on power estimation are following:

\section{E. Frequency Estimation}

Frequency is the most important parameter in power system monitoring, control, and protection. It can reflect the whole power system situation. In electrical power system it is of utmost importance to keep the frequency as close to its nominal value as possible. In order to control the power system frequency it needs to be measured quickly and accurately. But in general, power system voltage and current waveforms are distorted by harmonic and inter harmonic components, particularly during system disturbance. Faults or other switching transients may change the magnitude and phase angles of the waveforms. So the estimation of frequency is still an important and yet challenging part in power system. The effect of data length and SNR on MSE for frequency estimation is shown below:

\section{F. Effect of Data Length on MSE for Amplitude Power and Frequency Estimation}

The data sequence length influences the mean square error and therefore, the accuracy of high resolution methods depends on data length. The performance of the high resolution methods (Root MUSIC, MUSIC and ESPRIT) could be identified by comparing the mean square error of Amplitude, Power and Frequency estimation both for shorter and higher data length. Both for clean and noisy signal, the performance of the mean square error of amplitude, Power and Frequency estimation of the high resolution methods (Root MUSIC, MUSIC, and ESPRIT) are shown in Figure (1, 2, 3, 4, 5 and 6). When roughly summarizing different results from the Figure. (1, 2, 3, 4, 5 and 6) a list of data of Amplitude, Power and Frequency estimation both for clean and noisy signal in terms of MSE for increasing data length can be represented, as show that in Table (1, $2,3,4,5$ and 6$)$. 

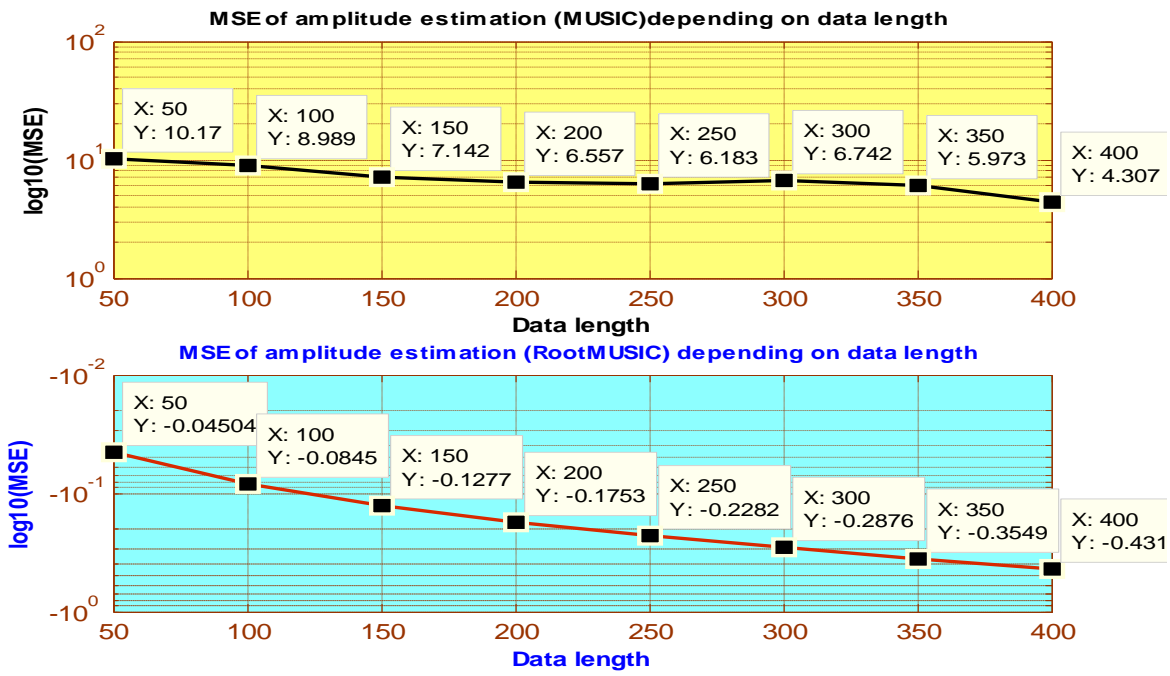

Fig. 1: Amplitude estimation (MUSIC \& Root MUSIC) in terms of MSE with respect to data length for clean signal in $\log$ scale.

Table 1: The amplitude estimation for clean signal in terms of MSE for increasing data length

\begin{tabular}{|c|c|c|}
\hline Data Length & MUSIC Method & Root MUSIC Method \\
\hline 50 & 10.17 & -0.04498 \\
\hline 100 & 8.989 & -0.08449 \\
\hline 150 & 7.142 & -0.12770 \\
\hline 200 & 6.557 & -0.17530 \\
\hline 250 & 6.183 & -0.22820 \\
\hline 300 & 6.742 & -0.28760 \\
\hline 350 & 5.973 & -0.35490 \\
\hline 400 & 4.307 & -0.43100 \\
\hline
\end{tabular}
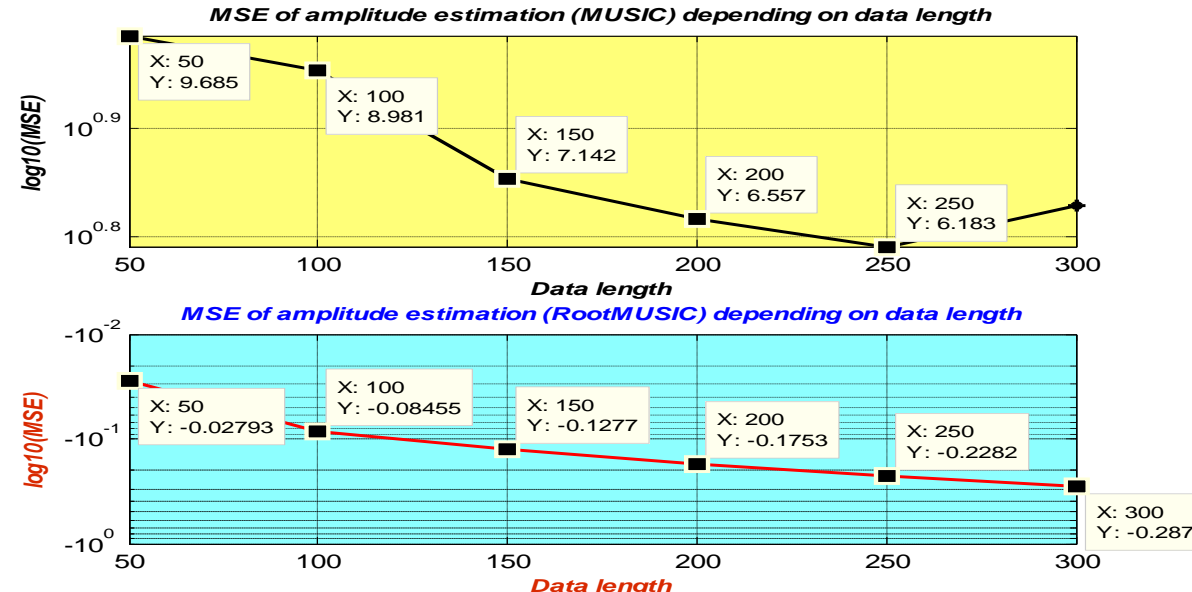

Fig. 2: Amplitude estimation (MUSIC \& Root MUSIC) for noisy signal in terms of MSE with respect to data length in log scale.

Table 2: The amplitude estimation for noisy signal in terms of MSE for increasing data length

\begin{tabular}{|c|c|c|}
\hline Data Length & MUSIC Method & Root MUSIC Method \\
\hline 50 & 9.685 & -0.04504 \\
\hline 100 & 8.981 & -0.08450 \\
\hline 150 & 7.142 & -0.12770 \\
\hline 200 & 6.557 & -0.17530 \\
\hline 250 & 6.183 & -0.22820 \\
\hline 300 & 6.742 & -0.28760 \\
\hline
\end{tabular}



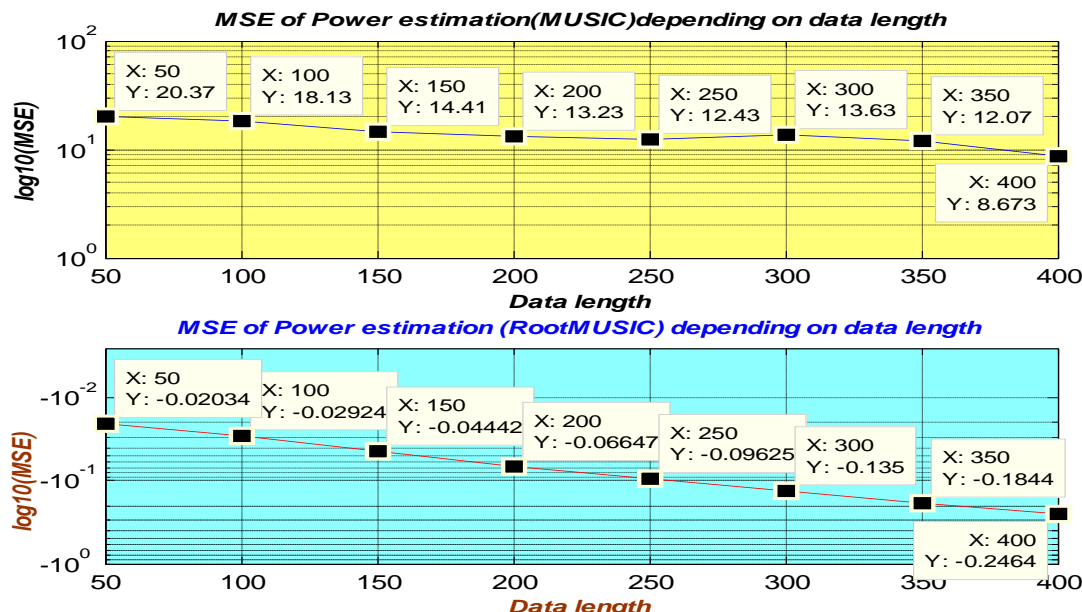

Fig. 3: Power estimation (MUSIC \& Root MUSIC) for clean signal in terms of MSE with respect to data length in log scale.

Table 3: The power estimation for clean signal in terms of MSE for increasing data length

\begin{tabular}{|c|c|c|}
\hline Data Length & MUSIC Method & Root MUSIC Method \\
\hline 50 & 20.39 & -0.02034 \\
\hline 100 & 18.13 & -0.02924 \\
\hline 150 & 14.41 & -0.04442 \\
\hline 200 & 13.23 & -0.06647 \\
\hline 250 & 12.43 & -0.09625 \\
\hline 300 & 13.63 & -0.13500 \\
\hline 350 & 12.07 & -0.18440 \\
\hline 400 & 8.673 & -0.24640 \\
\hline
\end{tabular}
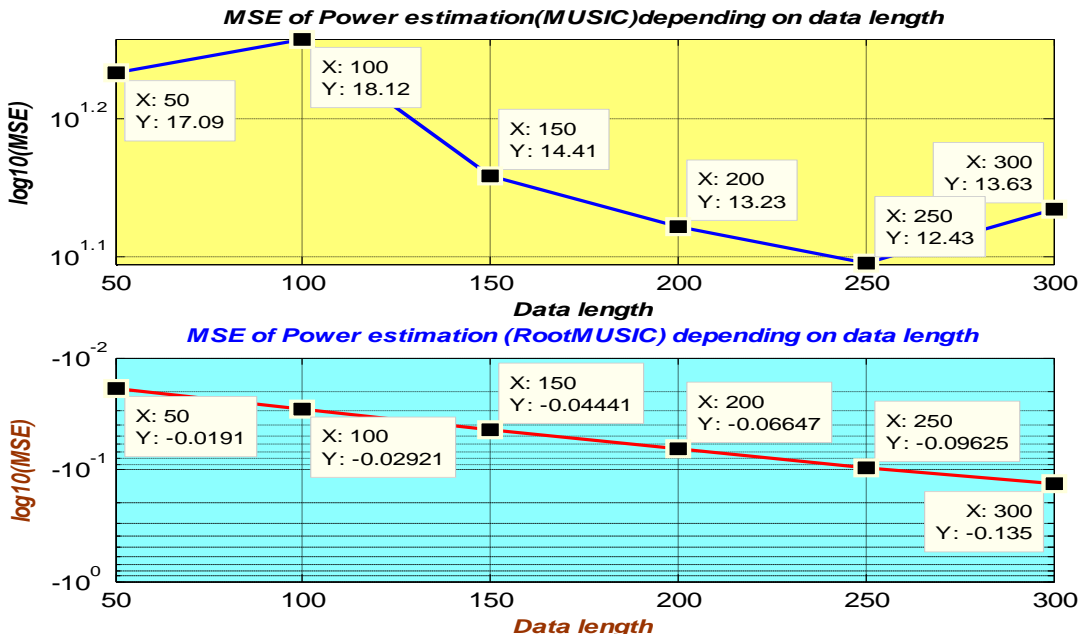

Fig. 4: Power estimation (MUSIC \& Root MUSIC) for noisy signal in terms of MSE with respect to data length in $\log$ scale.

Table 4: The power estimation for noisy signal in terms of MSE for increasing data length

\begin{tabular}{|c|c|c|}
\hline Data Length & MUSIC Method & Root MUSIC Method \\
\hline 50 & 17.09 & -0.01910 \\
\hline 100 & 18.12 & -0.02921 \\
\hline 150 & 14.41 & -0.04441 \\
\hline 200 & 13.23 & -0.06647 \\
\hline 250 & 12.43 & -0.09625 \\
\hline 300 & 13.63 & -0.13500 \\
\hline 350 & 5.973 & -0.35490 \\
\hline 400 & 4.307 & -0.39690 \\
\hline
\end{tabular}



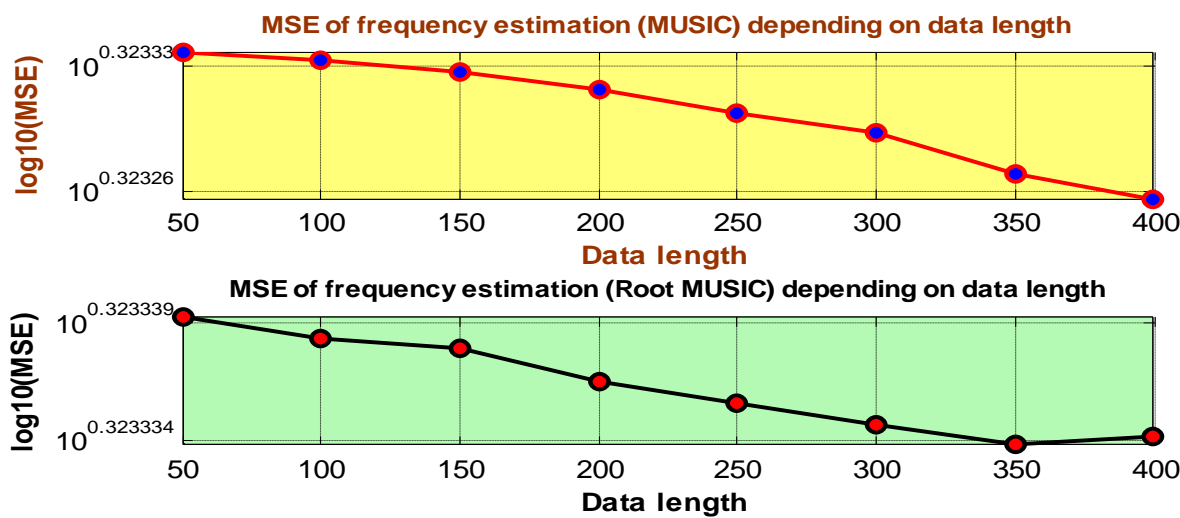

MSE of frequency estimation (ESPRIT) depending on data length

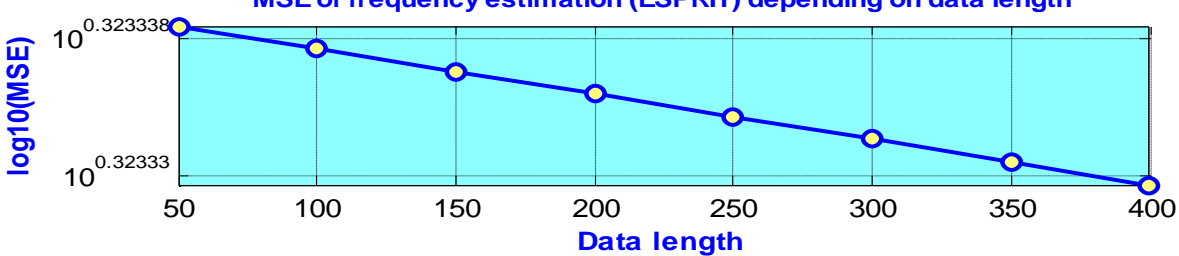

Fig. 5(a): Frequency estimation (MUSIC \& Root MUSIC) for clean signal in terms of MSE with respect to data length in $\log$ scale.

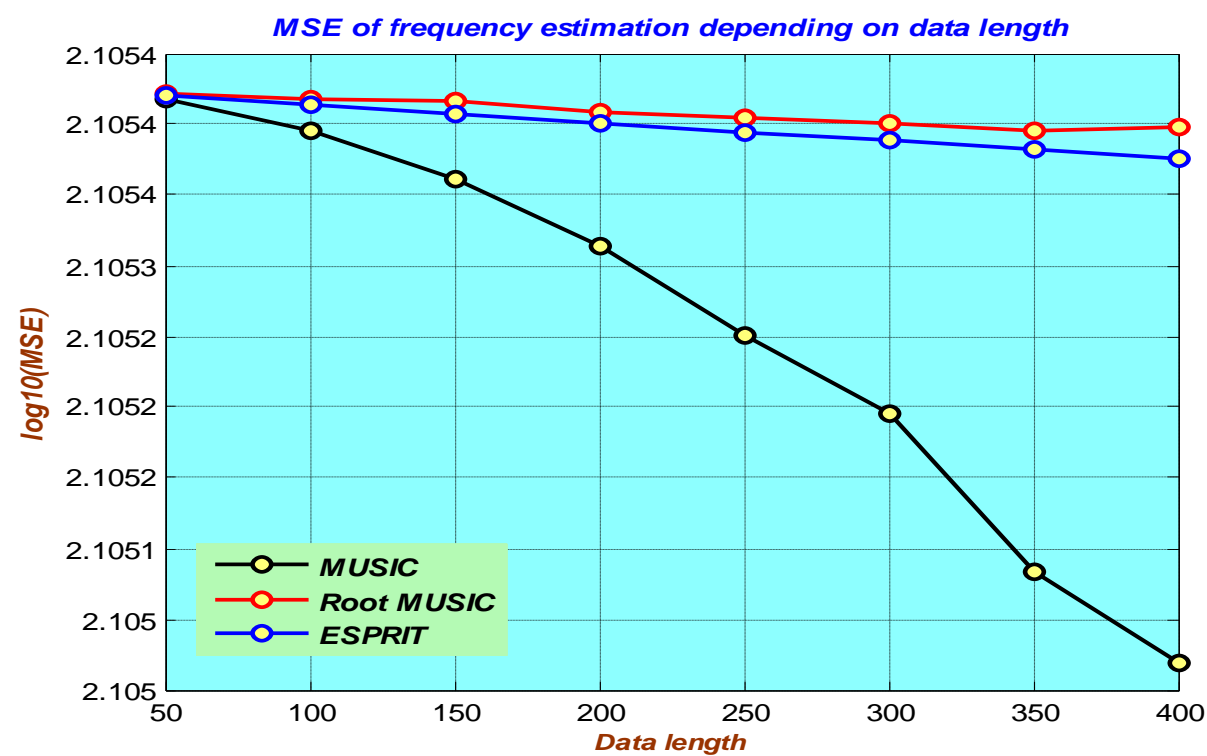

Fig. 5(b): Frequency estimation (MUSIC \& Root MUSIC) for clean signal in terms of MSE with respect to data length in linear scale.

Table 5: The Frequency estimation for clean signal in terms of MSE for increasing data length

\begin{tabular}{|c|c|c|c|}
\hline Data Length & MUSIC & Root MUSIC & ESPRIT \\
\hline 50 & 1.897 & 1.898 & 1.898 \\
\hline 100 & 1.896 & 1.898 & 1.898 \\
\hline 150 & 1.895 & 1.898 & 1.898 \\
\hline 200 & 1.891 & 1.898 & 1.898 \\
\hline 250 & 1.891 & 1.898 & 1.898 \\
\hline 300 & 1.886 & 1.898 & 1.898 \\
\hline 350 & 1.885 & 1.898 & 1.898 \\
\hline 400 & 1.883 & 1.898 & 1.898 \\
\hline
\end{tabular}



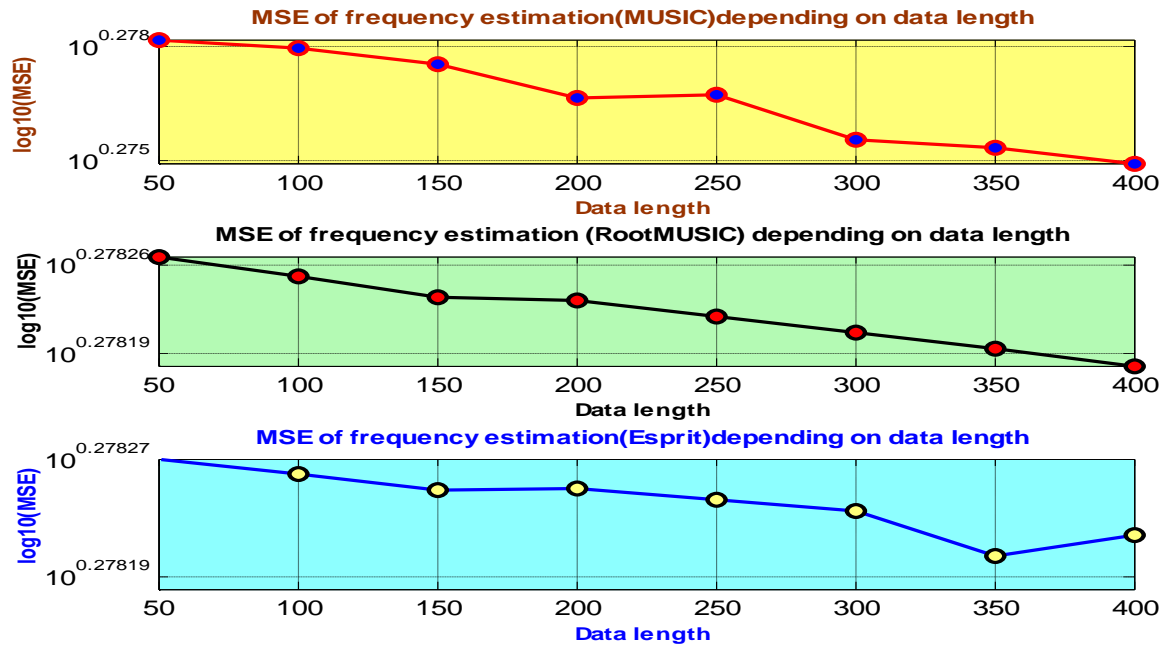

Fig. 6(a): Frequency estimation (MUSIC \& Root MUSIC) for noisy signal in terms of MSE with respect to data length in log scale.

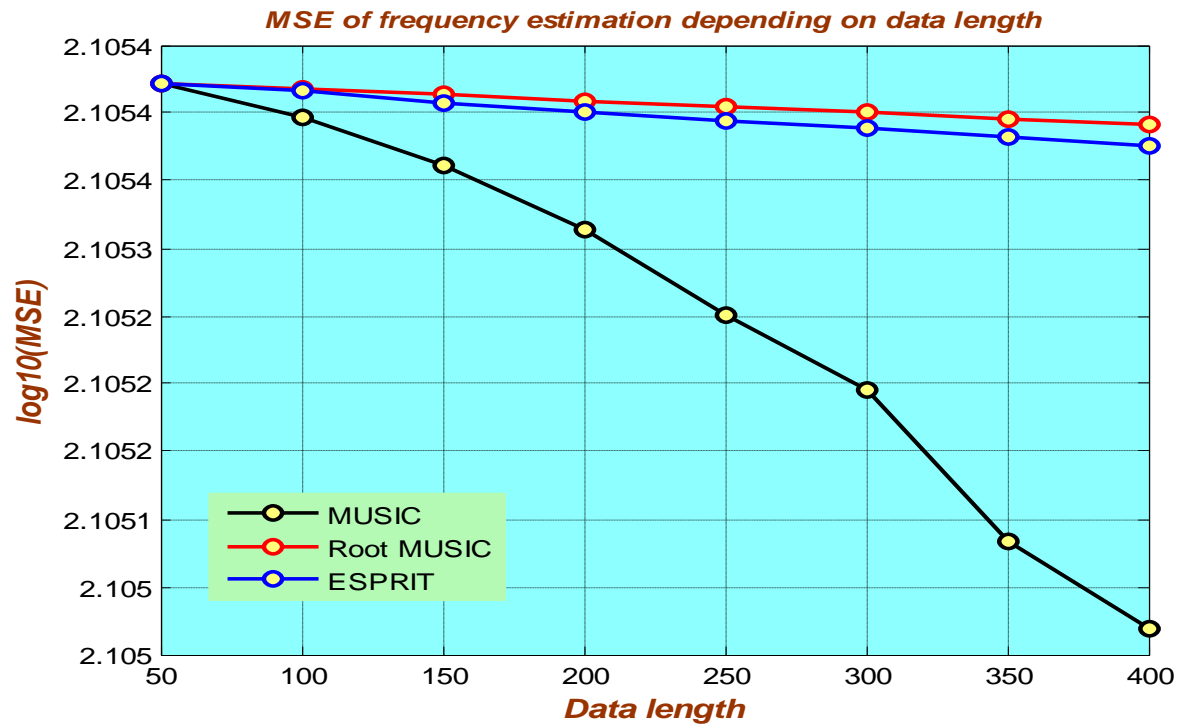

Fig. 6(b): Frequency estimation (MUSIC \& Root MUSIC) for noisy signal in terms of MSE with respect to data length in linear scale.

Table 6: The amplitude estimation for noisy signal in terms of MSE for increasing data length

\begin{tabular}{|c|c|c|c|}
\hline Data Length & MUSIC & ESPRIT & Root MUSIC \\
\hline 50 & 1.897 & 1.898 & 1.898 \\
\hline 100 & 1.896 & 1.898 & 1.898 \\
\hline 150 & 1.895 & 1.898 & 1.898 \\
\hline 200 & 1.891 & 1.898 & 1.898 \\
\hline 250 & 1.891 & 1.898 & 1.898 \\
\hline 300 & 1.886 & 1.898 & 1.898 \\
\hline 350 & 1.885 & 1.898 & 1.898 \\
\hline 400 & 1.883 & 1.898 & 1.898 \\
\hline
\end{tabular}

In Fig 1,2,3 and 4, it is seen that there is a sharp decrease of the estimation error for increasing length of the data sequence (pattern for MUSIC and Root MUSIC method results are similar). Root MUSIC method performs better for amplitude and power estimation in terms of MSE. In Fig 5 and Fig 6, it is seen that there is a sharp decrease of the estimation error for increasing length of the data sequence (pattern for MUSIC, Root MUSIC and ESPRIT method results are similar). Though it is seen that MUSIC method performs better for frequency estimation but for many simplifications, different assumptions and the complexity of the problem ESPRIT method is better than MUSIC and Root MUSIC. 


\section{G. Effect of SNR on MSE for Amplitude, Power and Frequency Estimation}

There is a strong dependency of the accuracy of the frequency estimation on SNR. The performance of the high resolution methods (MUSIC \& Root MUSIC) could be identified by comparing the mean square error of amplitude, power and frequency estimation both for very low and very high noise levels. Both for low and very high noise level the performance of the mean square error of amplitude estimation of the high resolution methods (MUSIC \& Root MUSIC and ESPRIT) are shown in Figure (1, 2, 3, 4, 5 and 6). When roughly summarizing different results from the Figure. (7,8 and 9) a list of data of Amplitude, Power and Frequency estimation both for low and very high noise level the performance of the mean square error can be represented, as show that in Table (7,8 and 9).
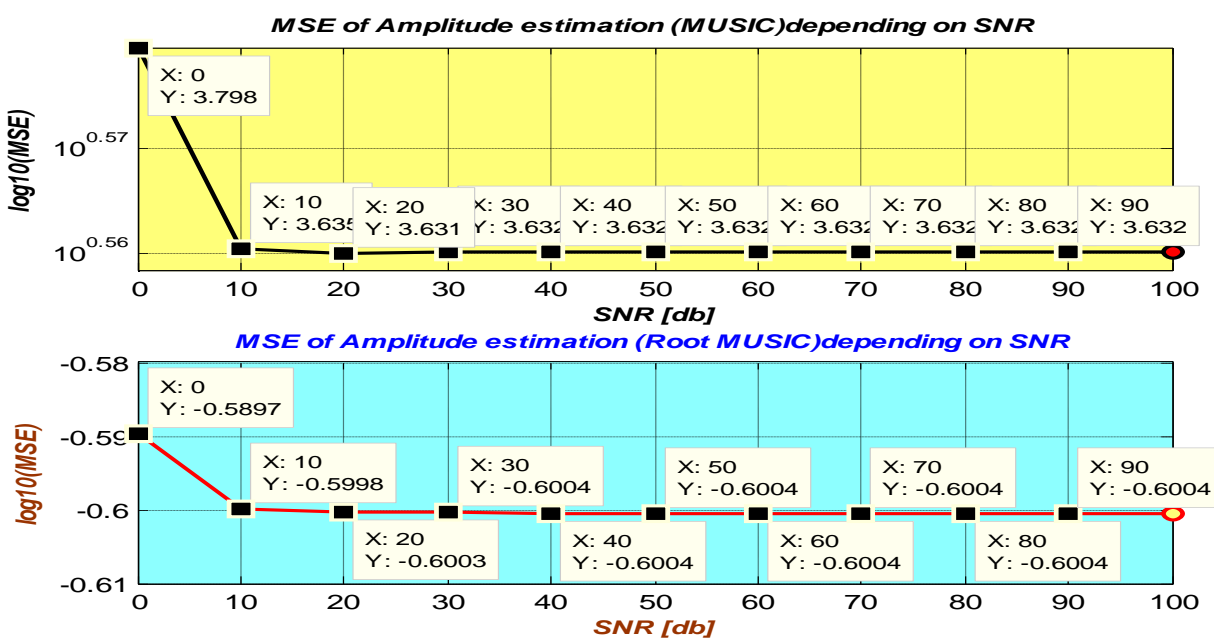

Fig. 7: Amplitude estimation (MUSIC \& Root MUSIC) in terms of MSE with respect to SNR in log scale.

Table 7: Amplitude estimation (MUSIC \& Root MUSIC) in terms of MSE for changing SNR

\begin{tabular}{|c|c|c|}
\hline Data Length & MUSIC Method & Root MUSIC Method \\
\hline 0 & 3.798 & -0.5897 \\
\hline 10 & 3.635 & -0.5998 \\
\hline 20 & 3.631 & -0.6003 \\
\hline 30 & 3.632 & -0.6004 \\
\hline 40 & 3.632 & -0.6004 \\
\hline 50 & 3.632 & -0.6004 \\
\hline 60 & 3.632 & -0.6004 \\
\hline 70 & 3.632 & -0.6004 \\
\hline 80 & 3.632 & -0.6004 \\
\hline 90 & 3.632 & -0.6004 \\
\hline 100 & 3.632 & -0.6004 \\
\hline
\end{tabular}
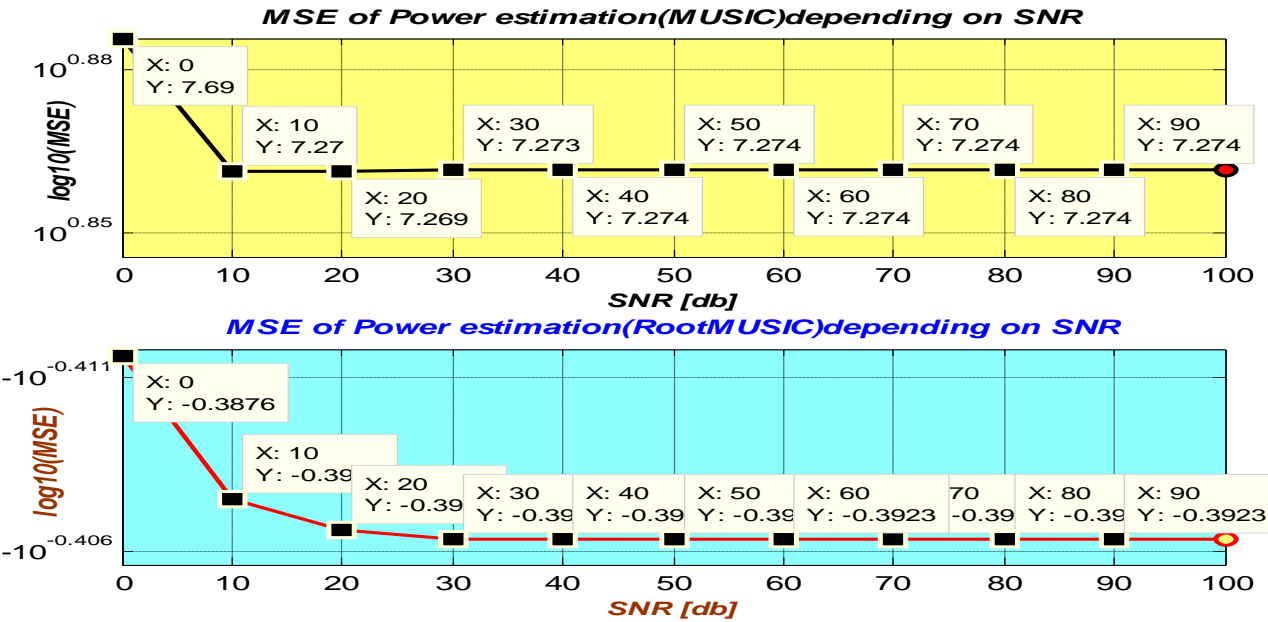

Fig. 8: Power estimation (MUSIC \& Root MUSIC) in terms of MSE with respect to SNR in log scale. 
Table 8: Power estimation (MUSIC \& Root MUSIC) in terms of MSE for changing SNR

\begin{tabular}{|c|c|c|}
\hline Data Length & MUSIC Method & Root MUSIC Method \\
\hline 0 & 7.690 & -0.3876 \\
\hline 10 & 7.270 & -0.3967 \\
\hline 20 & 7.273 & -0.3943 \\
\hline 30 & 7.274 & -0.3933 \\
\hline 40 & 7.274 & -0.3923 \\
\hline 50 & 7.274 & -0.3923 \\
\hline 60 & 7.274 & -0.3923 \\
\hline 70 & 7.274 & -0.3923 \\
\hline 80 & 7.274 & -0.3923 \\
\hline 90 & 7.274 & -0.3923 \\
\hline 100 & 7.274 & -0.3923 \\
\hline
\end{tabular}

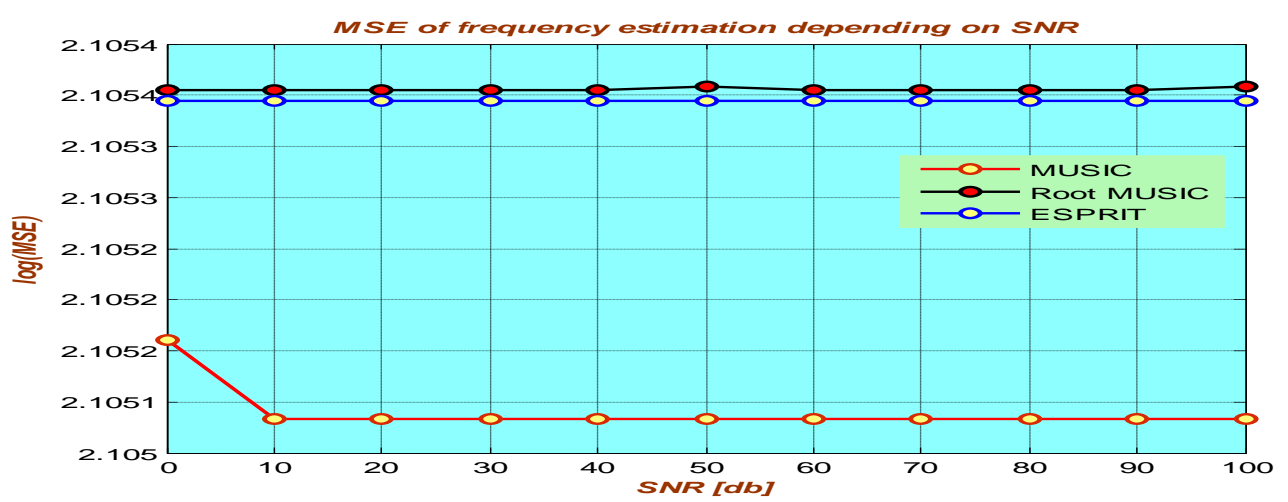

Fig. 9(a): Frequency estimation (MUSIC \& Root MUSIC) in terms of MSE with respect to SNR in log scale.
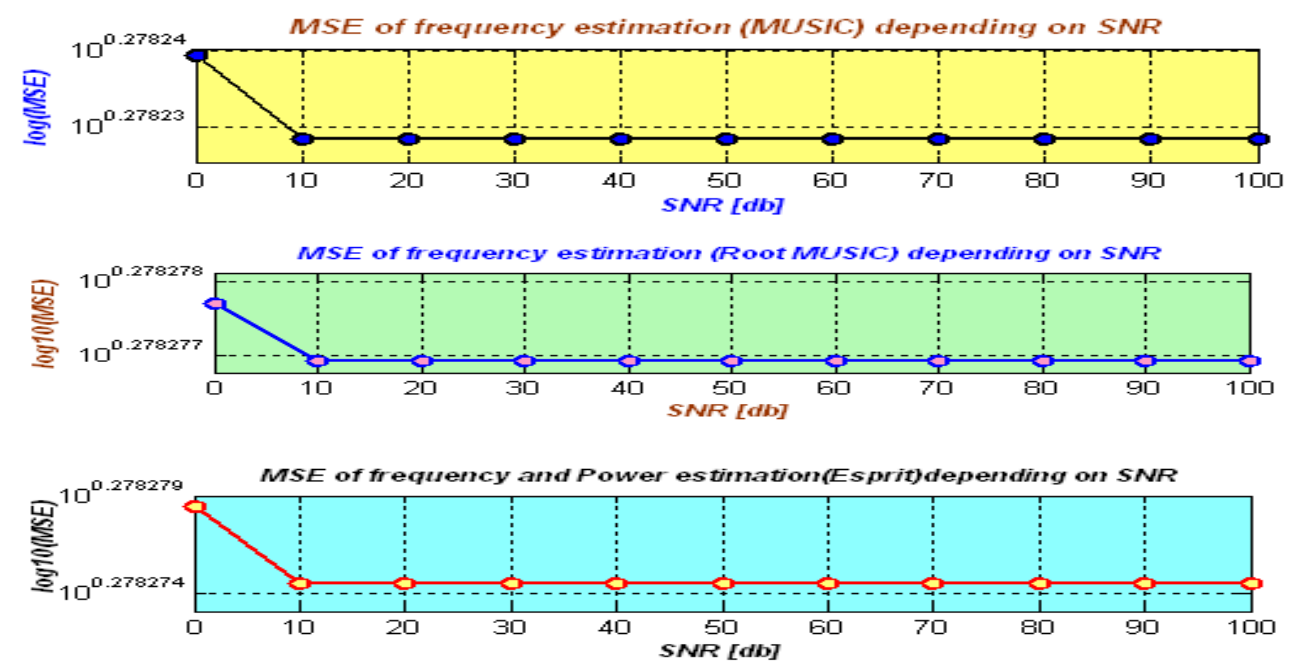

Fig. 9(b): Frequency estimation (MUSIC \& Root MUSIC) in terms of MSE with respect to SNR in linear scale.

Table 9: Frequency estimation (MUSIC \& Root MUSIC) in terms of MSE for changing SNR

\begin{tabular}{|c|c|c|c|}
\hline SNR & MUSIC Method & Root MUSIC Method & ESPRIT Method \\
\hline 0 & 2.1051 & 2.10543 & 2.10537 \\
\hline 10 & 2.1045 & 2.10541 & 2.10537 \\
\hline 20 & 2.1045 & 2.10541 & 2.10537 \\
\hline 30 & 2.1045 & 2.10541 & 2.10537 \\
\hline 40 & 2.1045 & 2.10541 & 2.10537 \\
\hline 50 & 2.1045 & 2.10541 & 2.10537 \\
\hline 60 & 2.1045 & 2.10541 & 2.10537 \\
\hline 70 & 2.1045 & 2.10541 & 2.10537 \\
\hline 80 & 2.1045 & 2.10541 & 2.10537 \\
\hline 90 & 2.1045 & 2.10541 & 2.10537 \\
\hline 100 & 2.1045 & 2.10541 & 2.10537 \\
\hline
\end{tabular}


In Fig 7 and Fig 8, it is seen that there is a sharp decrease of the estimation error for changing SNR (pattern for MUSIC and Root MUSIC method results are similar). Root MUSIC method performs better for amplitude and power estimation in terms of MSE. In Fig 9, it is seen that there is a sharp decrease of the estimation error for changing SNR (pattern for MUSIC, Root MUSIC and ESPRIT method results are similar). MUSIC method performs better for frequency estimation in terms of MSE.

\section{Conclusion}

The performance evaluation criterion and simulation results of the proposed methods are described in details. And the conclusion is that both methods (Root MUSIC \& ESPRIT) are similar in the sense that they are both eigen decomposition based methods which rely on decomposition of the estimated correlation matrix into two subspaces: noise and signal subspace. On the other hand, MUSIC uses the noise subspace to estimate the signal components while ESPRIT uses the signal subspace. In addition, the approach in many points is different. Root MUSIC method performs better for amplitude and power estimation in terms of MSE with respect to data length and SNR. Due to many simplifications, different assumptions and the complexity of the problem, simulation results represent the performance of ESPRIT method is better for frequency estimation in terms of MSE with respect to data length. Finally the performance of MUSIC method is better for frequency estimation with respect to SNR. The major contributions of this Paper are: Scopes for Future Works, In practical cases, it could be investigated and compared the performance of MUSIC, Root MUSIC and ESPRIT methods in terms of MSE with respect to the size of the correlation matrix. In practical cases, it could be investigated and compared the performance of amplitude estimation of MUSIC and ESPRIT in terms of MSE with respect to data length and SNR. The performance of amplitude, power and frequency estimation in terms of MSE of all the Methods mentioned above would be compared with respect to other high resolution methods.

\section{References}

[1] A. Bracale, G. Carpinelli, D Lauria, Z. Leonowicz, T. Lobos and J. Rezmer, "On Some Spectrum Estimation Methods for Analysis of Non-stationary Signals in Power Systems" - Part I:Theoretical aspects, Part II: Numerical Applications", Proc. 2004 11th International Conference on Harmonics and Quality of Power(ICHQP),

[2] A. Bracale, G. Carpinelli, T. Lobos, Z. Leonowicz and J. Rezmer,"Evaluation of compensation devices of DC arc furnaces using advanced spectrum estimation methods", Proc. 2005 15th Power Systems Computation Conference (PSCC),

[3] R.C.Dugan, M.F. McGranaghan, S. Santoso and H.W. Beaty, Electrical Power Systems Quality, 2nd. ed. McGraw-Hill, 2002.

[4] T.Lobs, Z.Leonowicz and J. Rezmer, "harmonics and interharmonics estimation using advanced signal processing methods" in proc. 9th IEEE Int. Conf. Harmonics and Quality of Power, Vol. I, Orlando, FL, 2000, pp. 335-340.

[5] S.M Kay, Modern Spectral Estimation: Theory and Application, Englewood Cliffs, NJ: Prentice-Hall, 1988, pp.224-225.

[6] T. Lobos, Z. Leonowicz, J. Rezmer and P. Schegner, "High-Resolution Spectrum Estimation Methods for Signal Analysis in Power Systems”, IEEE Trans. on Instr. and Meas., pp. 219-225, Feb. 2006

[7] R. carbone, D. Mennit, N. Sorrentino, and A.Testa, "Iterative harmonics and interharmonic analysis in multiconverter industrial systems,"in proc. 8th Int. Conf. Harmonics and Quality power, Athens,Greece,Oct.1998,PP.432-438

[8] A. Bracale, G.Carpinelli, D. Lauria, Z. Leonowiz, T. Lobos, and J. Rezmer, "On some spectrum estimation methods for analysis of non-stationary signals in power systems-Part II:Numerical applications,"in Proc. 11th Int. Conf. Harmonics and Quality Power, Lake Placid, NY, Sep. 2004,PP.6-16.

[9] R.C.Dugan, M.F. McGranaghan, S. Santoso and H.W. Beaty, Electrical Power Systems Quality, 2nd. ed. McGraw-Hill, 2002.

[10] R. Roy, T. Kailath, ESPRIT - Estimation of Signal Parameters via Rotational Invariance Techniques, IEEE Transactions on Acoustics, Speech, and Signal Processing, ASSP-37, 1989, 984-995.)

[11] S.H. Iaramillo, G.T. Heydt and E.O. Neill Carrello, "Power Quality Indices for Aperiodic Voltages and Currents", IEEE Trans.OnPower Delivery, pp. 784-790, Apr. 2000.

[12] C. W. Therrien, Discrete Random Signals and Statistical Signal Processing. EnglewoodCliffs, Prentice-Hall, 1992.

[13] Roland Priemer (1991). Introductory Signal Processing.WorldScientific.p.1.ISBN 9971509199.

[14] Some authors do not emphasize the role of information in the definition of a signal. For example, see PriyabrataSinha (2009). Speech processing in embedded systems. Springer.p. 9.ISBN 0387755802. "To put it very generally, a signal is any time-varying physical quantity.

[15] C.W. Therrien, Discrete Random Signals and Statistical Signal Processing (Englewood Cliffs, New Jersey,Prentice Hall PTR, 1992).

[16] K. V. S. Hari, B. V. Ramakrishnan, Performance analysis of modified spatial smoothing technique for direction estimation, Signal Processing, 79, 1999, 73-85. 